\title{
Pyruvate kinase M2 may be a therapeutic target in a mouse model of human non-small cell lung cancer
}

\author{
Akiko Suzuki ${ }^{*}$, Sachin Puri ${ }^{2}$, Bharat H Joshi ${ }^{1}$, Pamela Leland ${ }^{1}$, Bernard A Fox ${ }^{3}$, Raj K Puri ${ }^{1}$ \\ From 30th Annual Meeting and Associated Programs of the Society for Immunotherapy of Cancer (SITC 2015) \\ National Harbor, MD, USA. 4-8 November 2015
}

Non-small cell lung cancer (NSCLC) is the most common cause of cancer related mortality worldwide and in need of new treatment options. Previously, we have shown that 97 genes are overexpressed in 9 primary NSCLC cell lines by DNA microarray technology. Pyruvate kinase M2 (PKM2), an allosteric isoform of pyruvate kinase and metabolic enzyme necessary for aerobic glycolysis and cell proliferation, was identified to be over-expressed in the NSCLC cell lines. Real-time qRT-PCR and immunohistochemistry (IHC) techniques confirmed the microarrays results. Consistent with the over-expression of PKM2, all 9 NSCLC cell lines showed higher PKM2 enzyme activity than normal lung cell lines. Since a chemical inhibitor of PKM2 decreased PKM2 enzyme activity and proliferation of NSCLC cell lines, we hypothesized that PKM2 inhibitors may have a role in antitumor effects in vivo. Therefore, we evaluated antitumor effects of a PKM2 inhibitor in murine models of NSCLC. Mice tolerated intra-tumoral administration of the PKM2 inhibitor (Small molecule inhibitor for PKM2) up to $500 \mu \mathrm{g} / \mathrm{kg} /$ day without any evidence of visible toxicity or weight loss. Despite its anti-proliferative activities in vitro, the PKM2 inhibitor did not mediate significant antitumor effects in NSCLC tumor xenografts. The mechanism of lack of activity in vivo is not clear. It is possible that PMK2 inhibitor levels were not sufficient or sustained in vivo for a measurable antitumor effect.

To further study whether PKM2 was a target for therapy, we silenced the PKM2 gene in the H1299 and H358 NSCLC cell lines using RNAi and proliferative activity of tumors was assessed in vitro and in vivo. Gene silencing in cells transfected with plasmids encoding shRNAs was confirmed by IHC, which showed $>80 \%$ silencing measured by NIKON-S-Element software at the protein level. In clonogenic assays, we observed $>50 \%$ fewer colonies in two PKM2 silenced NSCLC cell lines compared to mock transfected controls. In subcutaneous xenograft tumor models in athymic nude mice, preliminary results showed that PKM2-silenced tumors grew significantly slower compared to mock transfected tumors $(\mathrm{p}<0.05)$. These results suggest that PKM2 may be a target for cancer therapy. Additionally, given its over-expression in NSCLC, it may also serve as a target for immunotherapy. Studies are ongoing to confirm these results, understand a mechanism of tumor response, and determine whether patients generate immune responses to PKM2.

\section{Authors' details}

${ }^{1}$ Center for Biologics Evaluation \& Research (CBER)/Food \& Drug Administration (FDA), Silver Spring, MD, USA. ${ }^{2}$ Molecular \& Tumor Immunology/Robert W. Franz Cancer Research Center/Earle A. Chiles Research Institute/Providence Cancer Center, Portland, OR, USA. ${ }^{3}$ Earle A. Chiles Research Institute, Portland, OR, USA.

Published: 4 November 2015

doi:10.1186/2051-1426-3-S2-P374

Cite this article as: Suzuki et al:: Pyruvate kinase M2 may be a therapeutic target in a mouse model of human non-small cell lung cancer. Journal for ImmunoTherapy of Cancer 2015 3(Suppl 2):P374. 